Article

\title{
Facile, One-Pot, Two-Step, Strategy for the Production of Potential Bio-Diesel Candidates from Fructose
}

\author{
Guo Qiu ${ }^{1}\left[10\right.$, Xincheng Wang ${ }^{2, *}$ (1) , Chongpin Huang ${ }^{1, *}$, Yingxia $\mathrm{Li}^{1}$ and Biaohua Chen ${ }^{1}$ \\ 1 State Key Laboratory of Chemical Resource Engineering, Beijing University of Chemical Technology, \\ Beijing 100029, China; qiuguo1994@163.com (G.Q.); liyx@mail.buct.edu.cn (Y.L.); \\ biaohuachen@yeah.net (B.C.) \\ 2 Beijing Key Laboratory of Fuels Cleaning and Advanced Catalytic Emission Reduction Technology, \\ Beijing Institute of Petrochemical Technology, Beijing 102617, China \\ * Correspondence: wxcnathan@gmail.com (X.W.); huangcp@mail.buct.edu.cn (C.H.); \\ Tel.: +86-10-8129-2131 (X.W.); +86-10-6441-2054 (C.H.)
}

Received: 23 June 2017; Accepted: 12 August 2017; Published: 17 August 2017

\begin{abstract}
The production of bio-diesel fuels from carbohydrates is a promising alternative to fossil fuels with regard to the growing severity of the environmental problem and energy crisis. Potential bio-diesel candidates or additives, such as 5-(hydroxymethyl)-2-(dimethoxymethyl) furan (HDMF), 2-(dimethoxymethyl)-5-(methoxymethyl) furan (DMMF), and 5-(methoxymethyl)-2-furaldehyde (MMF) could be produced from the alcoholic solutions of both 5-HMF and fructose in the presence of solid acid catalysts. In the present study, a readily prepared, silica, gel-supported nitric acid $\left(\mathrm{SiO}_{2}-\mathrm{HNO}_{3}\right)$ catalyst was found to be exceptionally reactive for the production of HDMF from fructose. A DMSO-methanol biphasic solvent system was developed and HDMF, DMMF, and MMF were observed at $150{ }^{\circ} \mathrm{C}$, with maximum yields of $34 \%, 34 \%$, and $25 \%$, respectively. Meanwhile, a maximum HDMF yield of 77\% was obtained from 5-HMF in methanol. Moreover, a sequential, one-pot, two-step dehydration/acetalization process, involving the dehydration of fructose to 5-HMF in dimethylsulfoxide (DMSO) at $150{ }^{\circ} \mathrm{C}$, and followed by adding a certain amount of methanol to react with the formed 5-HMF to HDMF at $100{ }^{\circ} \mathrm{C}$, was developed to promote the yield of HDMF. The optimum yield of HDMF reached $70 \%$ with the complete conversion of fructose. The reaction mechanisms of dehydration and acetalization have been proposed for the conversion of 5-HMF to HDMF. The two-step design allows for facile catalyst recycling while supplying as a promising method for the production of biodiesel from complex carbohydrates.
\end{abstract}

Keywords: bio-diesel; silica gel-supported nitric acid; one-pot two-step system

\section{Introduction}

Biomass represents an ideal substitute for fossil fuels and is considered as a potential renewable resource of energy and organic compounds [1,2]. Biodiesel can serve as an alternative fuel for diesel engines. 5-Hydroxymethylfurfural (5-HMF) is one of the most important biomass-derived platform compounds and can be used to produce various chemicals [3,4]. The acetals of 5-HMF have been obtained during the conversion of 5-HMF into derivatives with alcohols in acid mediums, including 5-(hydroxymethyl)-2-(dimethoxymethyl) furan (HDMF), 2-(dimethoxymethyl)-5-(methoxymethyl) furan (DMMF), and 5-(methoxymethyl)-2-furaldehyde (MMF); they are recognized as excellent additives for diesel due to their energy density and blending properties $[5,6]$.

Various alcohols (ethanol [7], isopropanol [8], and n-octanol [9]) have been screened for the reaction of 5-HMF and only tiny amounts of acetal products were detected. Moreover, it was found that both the conversion of 5-HMF and the yield of the corresponding acetals were low when using long-chain alcohols as reactants and solvents simultaneously, possibly due to long chain alcohols' 
strong adsorption ability on the catalyst [10]. Therefore, the short-chain alcohol methanol was employed as a reactant during the acetalization of 5-HMF to reduce the passivation rate of the catalyst and allow easy removal of the solvent.

It was reported that HDMF, DMMF, and MMF were important intermediates during the formation of methyl levulinate from the esterification of bio-oil components with methanol over Amberlyst 70 [11]. Particularly, HDMF can be used to produce levulinic acid and its derivatives [12], which are also described as potential fuel additives or bio-diesel fuel. The acetalization of 5-HMF with $\mathrm{MeOH}$ was performed at $65^{\circ} \mathrm{C}$ by Arias et al. [10] using $1.5 \mathrm{NaBeta}$ zeolite, and 99\% selectivity of HDMF with 99\% conversion of 5-HMF was obtained after $2.5 \mathrm{~h}$, indicating that appropriate control of the catalyst acidity was beneficial for the acetalization reaction. Balakrishnan et al. [7] attempted to employ silica sulfuric acid as a catalyst in the synthesis of ethanol acetals of 5-HMF, and a 36\% yield of 5-(ethoxy methyl) furfural from 5-HMF was obtained at $75{ }^{\circ} \mathrm{C}$. Compared with 5-HMF, fructose represents a more economically available substrate. Lai et al. [8] reported that 5-HMF-derived furfurals (MMF, HDMF, and DMMF) could be obtained from fructose using $\mathrm{HCl}$ in methanol at $80^{\circ} \mathrm{C}$ with a reaction time of $8 \mathrm{~h}$; however, only $25 \%$ total yields were obtained. However, there is a lack of a highly efficient system for the production of 5-HMF-derived acetals from fructose. Therefore, a one-pot dehydration/acetalization process starting from fructose was investigated in the present study.

Silica gel has a large surface area and an open pore structure, and it is stable, non-toxic, highly active, and can act as an amorphous porous adsorbent material [13]; therefore, it is often used as a carrier in industrial catalysis. In this study, silica gel-supported $\mathrm{HNO}_{3}\left(\mathrm{SiO}_{2}-\mathrm{HNO}_{3}\right)$ was used for the dehydration/acetalization reaction of 5-HMF; in this way, the active sites were grafted onto silica gel, giving them the advantages of ready availability, readily prepared procedure, long catalytic life, environmental-friendliness, good to excellent yields, and recyclability [14].

With the aim of producing HDMF from fructose efficiently via a green route, the work was designed in three parts: firstly, the acetalization of 5-HMF with methanol; secondly, fructose as the substrate to produce HDMF directly in a DMSO-methanol biphasic solvent system; thirdly, a novel, one-pot, two-step strategy involving the addition of methanol after the reaction of fructose in DMSO. In this way, the production of HMDF could be greatly promoted, and this method can serve as an efficient approach for the production of other promising chemicals from carbohydrates.

\section{Results and Discussion}

\subsection{Production of HDMF in Methanol Solvent}

Due to the bifunctionality of 5-HMF, a large number of competitive reactions such as oxidation, etherification, and polymerization might occur in the system [15]; therefore, the use of a selective catalyst to promote the acetalization of 5-HMF is an essential part of the research. The production of HDMF and DMMF from 5-HMF and fructose in methanol were performed to investigate the behavior of $\mathrm{SiO}_{2}-\mathrm{HNO}_{3}$. It was found that the formation of $\mathrm{HDMF}$ and DMMF were favored during the reaction of 5-HMF over $\mathrm{SiO}_{2}-\mathrm{HNO}_{3}$ in methanol. The highest yield of $\mathrm{HDMF}$ was up to $77 \%$ at $60{ }^{\circ} \mathrm{C}$ (as shown in Figure 1a). It was proposed that the presence of hydroxyl groups on the surface of the $\mathrm{SiO}_{2}-\mathrm{HNO}_{3}$, leading to a relatively high concentration of $\mathrm{H}^{+}$on the surface of catalysts, greatly promoted the acetalization of 5-HMF with methanol. Only trace amounts of DMMF and MMF were observed. However, at an elevated temperature $\left(120^{\circ} \mathrm{C}\right)$, the yields of DMMF and MMF reached $80 \%$ and $7 \%$, respectively, indicating further reactions over the -OH group of dimethyl acetal and 5-HMF (Figure 1b). Therefore, at higher temperatures, the -OH groups of both 5-HMF and HDMF could be attacked and etherified by methanol, and the leading reaction in the system was the etherification of the -OH group because a portion of 5-HMF was converted directly to MMF, while the HDMF reacted further into DMMF (as illustrated in Scheme 1). This result indicates that the etherification reaction requires higher temperature than acetalization, suggesting that low temperatures are recommended for the selective 
production of HDMF from 5-HMF. It is important to note that the formation of brownish black humins was also observed at high temperatures, as has been reported in similar reactions [16].

(a)

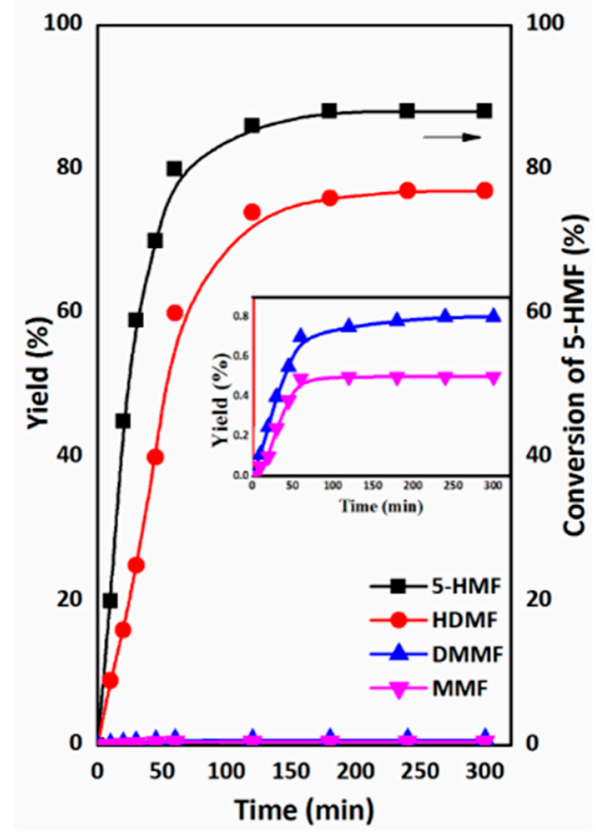

(b)

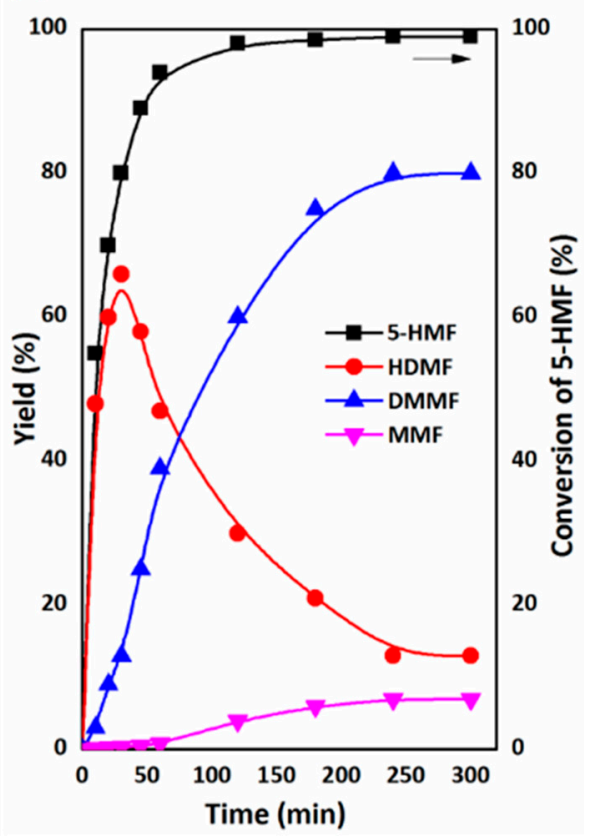

Figure 1. Acetalization of 5-Hydroxymethylfurfural (5-HMF) in methanol. (a) $0.77 \mathrm{mmol} 5-\mathrm{HMF}$ in $4.0 \mathrm{~mL}$ of methanol at $60{ }^{\circ} \mathrm{C}$ with $26 \mathrm{mg}$ of $\mathrm{SiO}_{2}-\mathrm{HNO}_{3}$. (b) $0.77 \mathrm{mmol} 5-\mathrm{HMF}$ in $4.0 \mathrm{~mL}$ of methanol at $120{ }^{\circ} \mathrm{C}$ with $26 \mathrm{mg}$ of $\mathrm{SiO}_{2}-\mathrm{HNO}_{3}$. (Inset: details for 5-HMF, 5-(hydroxymethyl)-2-(dimethoxymethyl) furan (HDMF), 2-(dimethoxymethyl)-5-(methoxymethyl) furan (DMMF), and 5-(methoxymethyl)-2-furaldehyde (MMF) with yields in the range from $0 \%$ to $0.8 \%$ ).

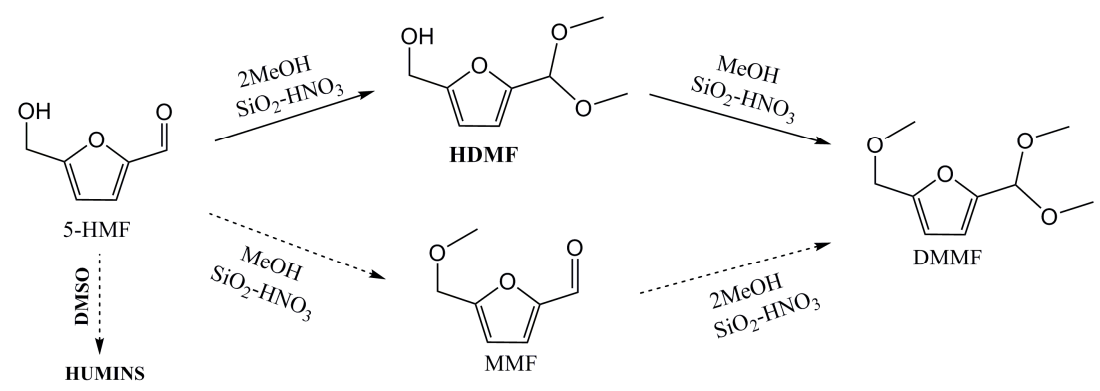

Scheme 1. Acetalization of 5-HMF in methanol using $\mathrm{SiO}_{2}-\mathrm{HNO}_{3}$.

Using fructose as a substrate, $\mathrm{SiO}_{2}-\mathrm{HNO}_{3}$ was expected to enhance both the dehydration of fructose and the production of HDMF, as both reactions have been reported to be promoted by acid catalysts [17]. Nevertheless, the maximum yields of HDMF and DMMF were only $3 \%$ and $8 \%$, respectively (Table 1), showing that the solid catalyst has limited catalytic activity for the direct conversion of fructose to HDMF in methanol. According to previous studies, the solvent has a strong influence on the dehydration reaction and plays an important role in the formation of the intermediate compounds that lead to the production of 5-HMF [18]. Furthermore, using methanol as a medium and no catalyst led to the formation of anhydrosugars, mainly including methyl fructofuranoside and methyl D-fructopyranoside, according to Liu [19]. However, the production of 5-HMF, MMF, and methyl levulinate has been achieved in the presence of sulphuric acid [20], proving that the 
introduction of the appropriate catalyst can change the product distribution and lead to the synthesis of 5-HMF.

Table 1. Results of the reaction of fructose and methanol in the presence of $\mathrm{SiO}_{2}-\mathrm{HNO}_{3} \cdot{ }^{a}$

\begin{tabular}{|c|c|c|c|c|c|c|}
\hline $\begin{array}{l}\text { Temp. } \\
\left({ }^{\circ} \mathrm{C}\right)\end{array}$ & $\begin{array}{c}\text { Time } \\
\text { (h) }\end{array}$ & $\begin{array}{c}\text { Fructose Conversion } \\
(\%)\end{array}$ & $\begin{array}{c}\text { 5-HMF Yield } \\
(\%)\end{array}$ & $\begin{array}{l}\text { HDMF Yield } \\
(\%)\end{array}$ & $\begin{array}{c}\text { DMMF Yield } \\
(\%)\end{array}$ & $\begin{array}{c}\text { MMF Yield } \\
(\%)\end{array}$ \\
\hline \multirow{3}{*}{80} & 4 & 98 & 0 & 1.1 & 0 & 0 \\
\hline & 8 & 98 & 0 & 3 & 1 & 0 \\
\hline & 24 & 100 & 2 & 1 & 5 & 1 \\
\hline \multirow{3}{*}{100} & 4 & 99 & 1 & 2 & 4 & 0 \\
\hline & 8 & 99 & 2 & 3 & 4 & 0 \\
\hline & 24 & 100 & 3 & 1 & 6 & 6 \\
\hline \multirow{3}{*}{120} & 4 & 99 & 2 & 1.3 & 3 & 4 \\
\hline & 8 & 100 & 4 & 1 & 8 & 6 \\
\hline & 24 & 100 & 6 & 2 & 6 & 8 \\
\hline
\end{tabular}

${ }^{a}$ Reaction Conditions: $1.37 \mathrm{mmol}$ fructose in $4.0 \mathrm{~mL}$ methanol with $50 \mathrm{mg} \mathrm{SiO} 2-\mathrm{HNO}_{3}$.

Additionally, it is essential to remark on the role of water in the system, as water has an essential impact on both the dehydration and acetalization reactions, according to Shimizu [21]. For the HDMF, DMMF, and MMF molecules, 4, 5, and 4 water molecules are decomposed from fructose, respectively, not only affecting the rehydration of 5-HMF to levulinate acid, but also decreasing the maximum attainable HDMF concentration due to the reversible nature of the acetalization reaction.

With the aim of studying the reaction process, a first-order rate equation for the fructose dehydration in methanol was reported, obtaining a total rate constant of approximately $1 \times 10^{-6} \mathrm{~s}^{-1}$ for the competing reaction paths [18], and hence small fractions of 5-HMF and its derivatives can be detected under the influence of the $\mathrm{SiO}_{2}-\mathrm{HNO}_{3}$ catalyst. Due to the nature of the sequential reactions in the system, the low formation rate of 5-HMF affects the overall performance, but with longer reaction times, the overall conversion towards 5-HMF and its derivatives was sped up, thus favoring the formation of DMMF and MMF (Table 1).

Moreover, it is important to note that methyl levulinate was also detected but not quantified, and no change of colour in the reaction mixture was observed, indicating little or no formation of humins.

\subsection{Production of HDMF in a DMSO/Methanol Biphasic System}

Due to the low 5-HMF yield from fructose in methanol, a biphasic solvent system (with various volume ratios of DMSO and methanol) was utilized in a one-pot system so that the dehydration of fructose and the subsequent acetalization of 5-HMF could be achieved by $\mathrm{SiO}_{2}-\mathrm{HNO}_{3}$ simultaneously. The effect of DMSO on the reaction was studied, and the results were similar to the ones reported for the production of other 5-HMF derivatives in DMSO (Figure 2) [22]. The addition of DMSO did not necessarily affect the conversion of fructose but instead changed the distribution of the products, leading to the production of 5-HMF.

The addition of DMSO, even at a low ratio, improved the 5-HMF formation and, in the same way, the consecutive acetalization and etherification reactions. Figure 3 shows that the yields of 5-HMF, HDMF and DMMF increased with increasing DMSO addition at the initial stages. The reaction rate of the acetalization reaction of 5-HMF was higher than the rate of the dehydration reaction of fructose when the DMSO content is within $30 \mathrm{vol} \%$, suggesting that the high methanol concentration in the system favors HDMF formation. Nevertheless, with further increases in DMSO concentration, the dehydration of fructose to 5-HMF becomes the main reaction, possibly because the low methanol content was not sufficient for the subsequent reactions. 


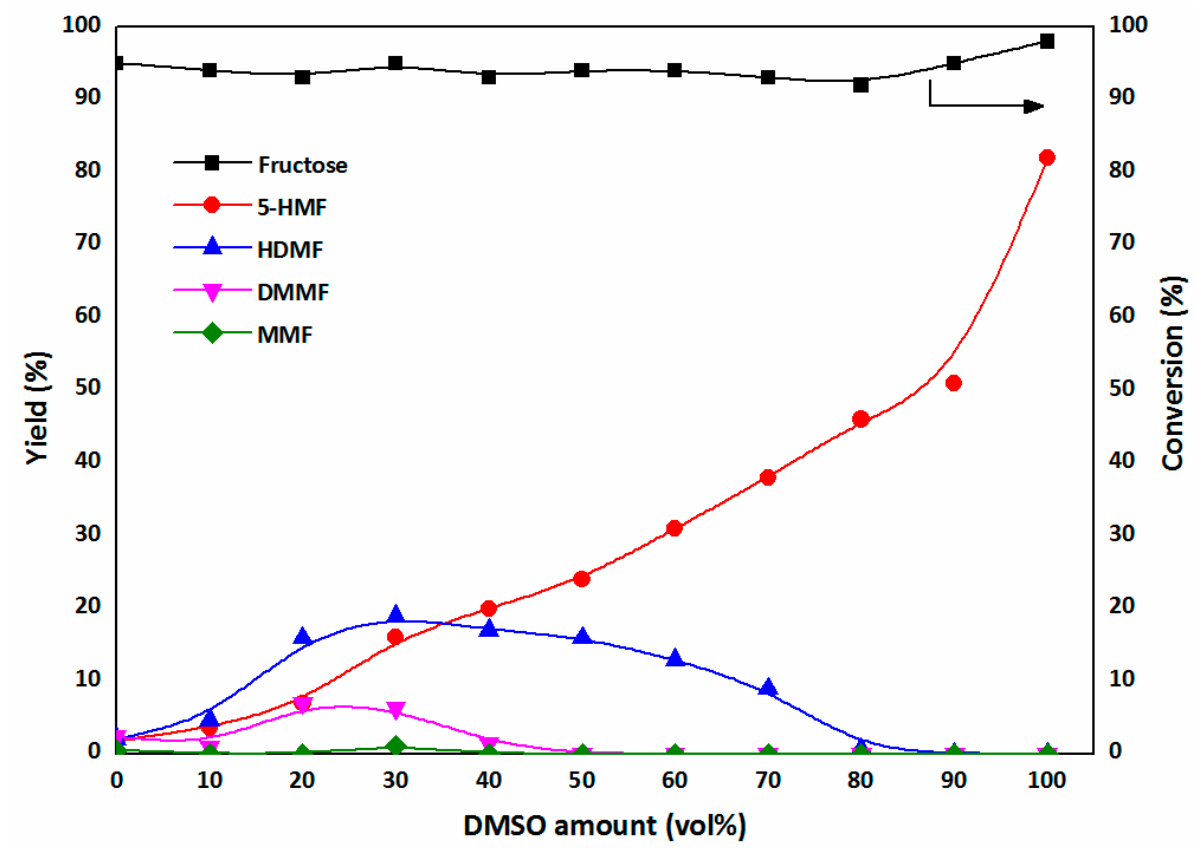

Figure 2. Effect of dimethylsulfoxide (DMSO) content on the reaction. Reaction conditions: $150{ }^{\circ} \mathrm{C}, 2 \mathrm{~h}$, $1.37 \mathrm{mmol}$ fructose, $50 \mathrm{mg} \mathrm{SiO} 2-\mathrm{HNO}_{3}, 4.0 \mathrm{~mL}$ solvent.

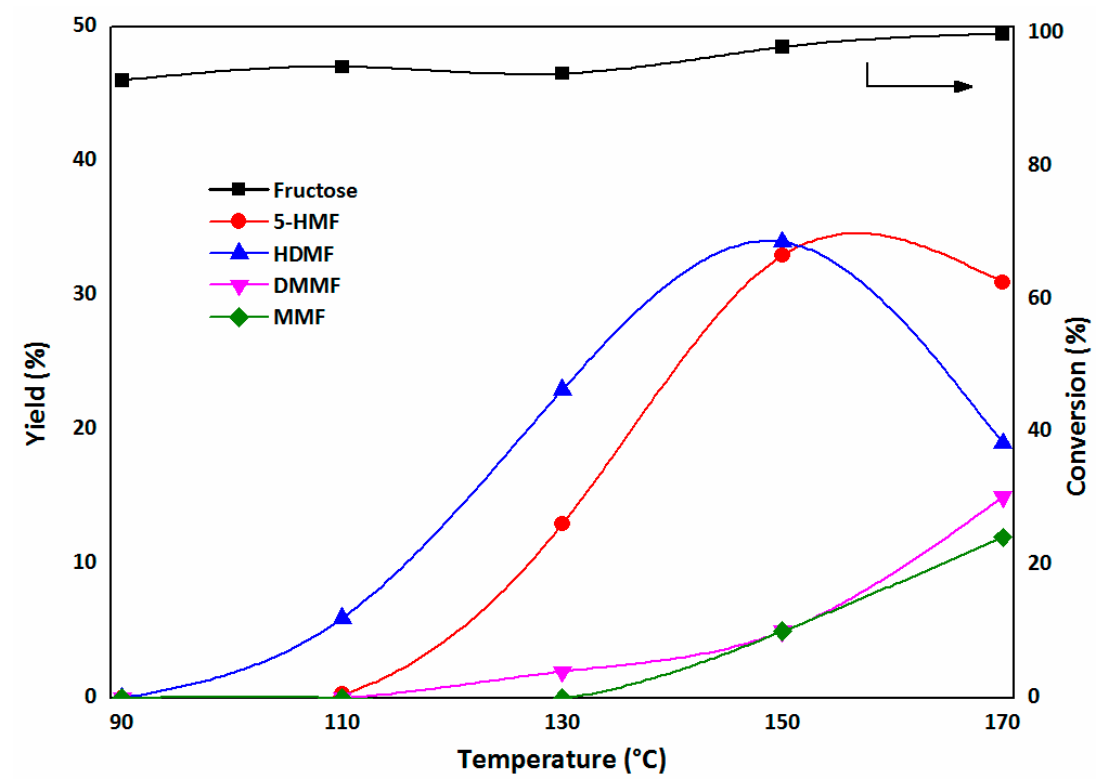

Figure 3. Effect of temperature on the reaction. Reaction conditions: $30 \mathrm{~min}, 1.37 \mathrm{mmol}$ fructose, $50 \mathrm{mg}$ $\mathrm{SiO}_{2}-\mathrm{HNO}_{3}, 4.0 \mathrm{~mL}$ solvent (DMSO/methanol 30/70 v/v).

The optimal volume ratio of DMSO/methanol (30/70) was utilized to continue further research for the production of HDMF. It is worth mentioning that despite the increase in the concentration of 5-HMF and its derivatives, the optimum yield of HDMF from fructose is less than 50\%, and methanol has a strong inhibitory effect on the formation of 5-HMF, leading to the production of HDMF.

To better understand the effect of the temperature on the reaction, a series of reactions was conducted at various temperatures. As shown in Figure 3, at low temperatures, the formation of 5-HMF was slight or almost null; therefore, further acetalization or etherification reactions of 5-HMF were not possible, indicating that the initial reaction stage is the key step in the process. It has been 
reported that fructose dehydration using DMSO could be promoted at temperatures above $140{ }^{\circ} \mathrm{C}$ [23], which also promoted the reactions to form DMMF and MMF. Figure 3 depicts a typical behavior of a consecutive reaction system, showing that the maximum HDMF concentration was reached at $150{ }^{\circ} \mathrm{C}$. Upon further increasing the temperature, both the etherification of the dimethyl acetal of 5-HMF and the formation of humins were facilitated [22].

The behavior of the reaction with different reaction times at $150{ }^{\circ} \mathrm{C}$ is presented in Figure 4. High fructose conversion (almost 98\%) was achieved within $30 \mathrm{~min}$. Meanwhile, the 5-HMF yield reached a maximum at $35 \%$ and then declined gradually. An optimum HDMF yield of $34 \%$ was obtained after $30 \mathrm{~min}$. As the reaction time was prolonged, the formation of DMMF and MMF, as well as humins, increased along with the decrease of 5-HMF and HDMF. High temperatures and short reaction times provided the optimized reaction conditions for the formation of HDMF, since under these conditions it was possible to improve the dehydration rate of fructose to 5-HMF, which was found to be the bottleneck of the system. With a short reaction period, it is possible to avoid excessive increases in the production of both DMMF and MMF. Long reaction times were detrimental to HDMF formation; therefore, 30 min was taken as the optimal reaction time for further study.

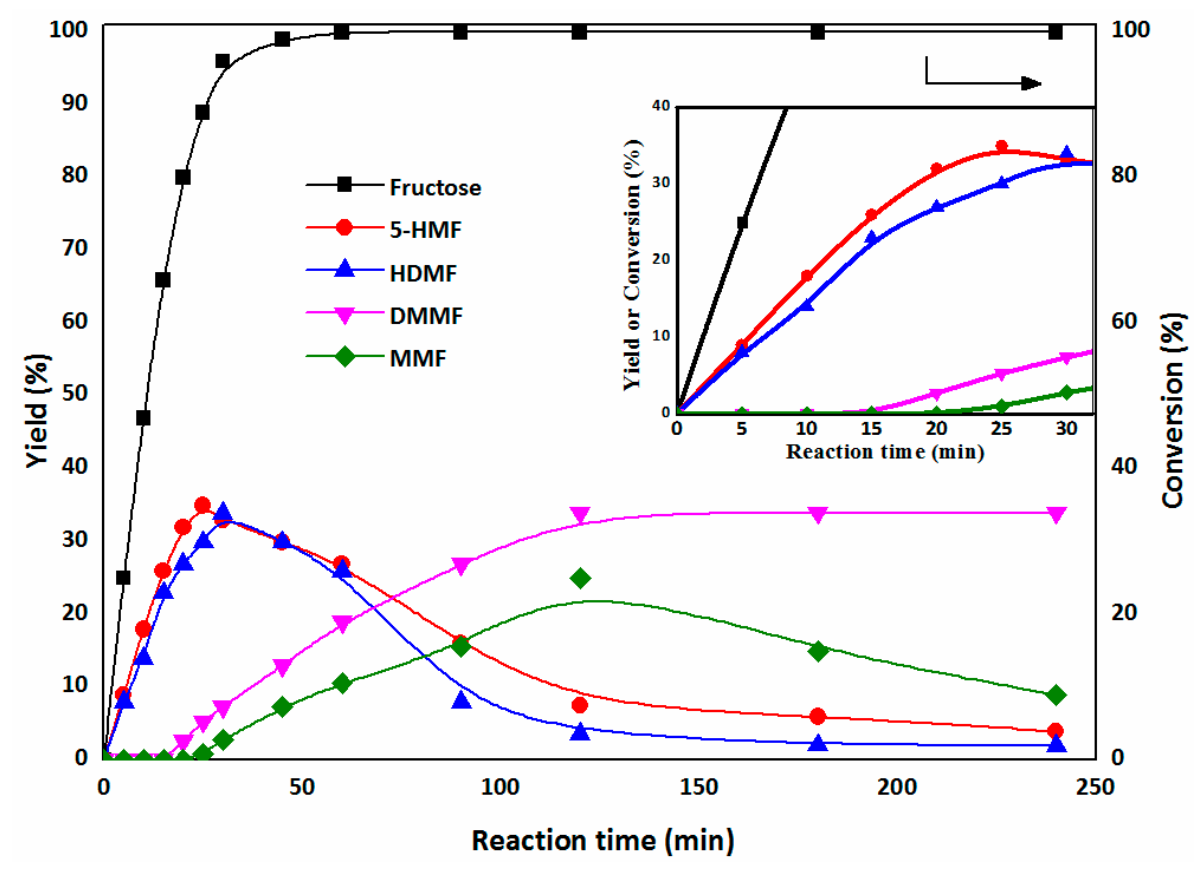

Figure 4. Effect of reaction time on the reaction. Reaction conditions: $150{ }^{\circ} \mathrm{C}, 1.37 \mathrm{mmol}$ fructose, $50 \mathrm{mg}$ $\mathrm{SiO}_{2}-\mathrm{HNO}_{3}, 4.0 \mathrm{~mL}$ solvent (DMSO/methanol 30/70 v/v). (Inset: details for 5-HMF, HDMF, DMMF, and MMF for the first $30 \mathrm{~min}$ of the reaction).

The effect of catalyst amount on the dehydration of fructose and the following further acetalization and etherification of 5-HMF was investigated (Figure 5). In the absence of $\mathrm{SiO}_{2}-\mathrm{HNO}_{3}$, neither 5-HMF nor other products were detected despite a fructose conversion of $91 \%$, confirming the important role of $\mathrm{SiO}_{2}-\mathrm{HNO}_{3}$. The addition of $\mathrm{SiO}_{2}-\mathrm{HNO}_{3}$ significantly changed the behavior of the reaction by prompting the production of 5-HMF as well as the subsequent acetalization to HDMF. Increasing the amount of $\mathrm{SiO}_{2}-\mathrm{HNO}_{3}$ led to the production of HDMF, DMMF, and MMF up to maximum yields of $34 \%, 10 \%$, and $18 \%$, respectively. Nevertheless, a substantial color change of the reaction mixture was observed upon further increasing the $\mathrm{SiO}_{2}-\mathrm{HNO}_{3}$ amount, indicating the appearance of insoluble products. Moreover, a drastic change in the product distribution with 5-HMF as the main product in the system was observed, and its etherification towards MMF was a preferred consecutive reaction. 


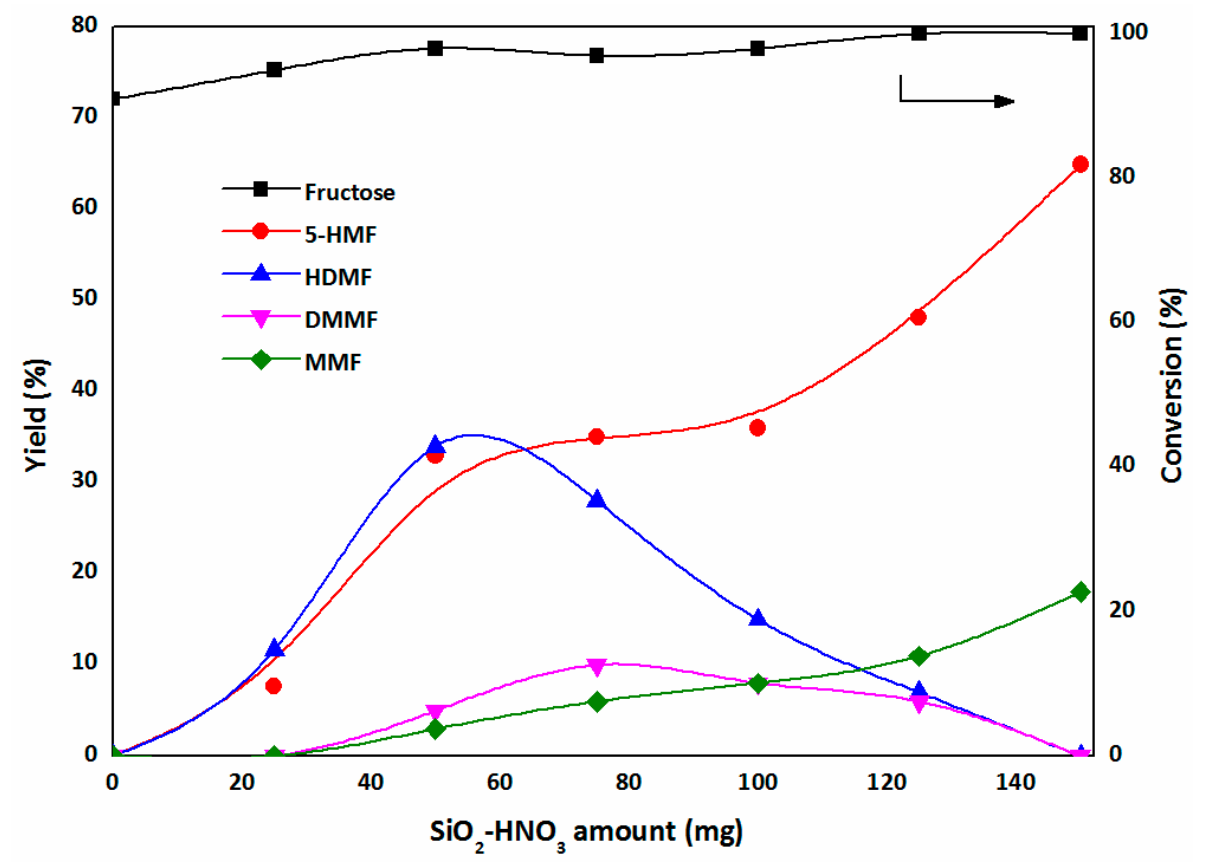

Figure 5. Effect of catalyst $\mathrm{SiO}_{2}-\mathrm{HNO}_{3}$ amount on the reaction. Reaction conditions: $150{ }^{\circ} \mathrm{C}, 30 \mathrm{~min}$, $1.37 \mathrm{mmol}$ fructose, $4.0 \mathrm{~mL}$ solvent (DMSO/methanol 30/70 v/v).

It was obvious that the overloading of $\mathrm{SiO}_{2}-\mathrm{HNO}_{3}$ beyond $50 \mathrm{mg}$ was unfavorable for the acetalization of 5-HMF, represented by the decreased concentration of HDMF and its derivative DMMF. The disappearance of DMMF may be explained by the polymerization reaction leading to the formation of humins. The etherification reaction of 5-HMF to MMF was promoted by high catalyst dosage, possibly due to the catalyst's effect or the high 5-HMF concentration.

Obviously, this complicated reaction system requires an appropriate amount of catalyst to enhance the production of HDMF and avoid the formation of side-products, and the optimal amount of $\mathrm{SiO}_{2}-\mathrm{HNO}_{3}$ was found to be $50 \mathrm{mg}$.

\subsection{One-Pot, Two-Step Design for the Conversion of Fructose into HDMF}

To improve the production of HDMF from fructose, the dehydration of fructose to 5-HMF plays an important role, and the higher reaction temperature might lead to further dehydration or polymerization of 5-HMF to unwilling byproducts. Therefore, the consecutive reactions should be designed properly to favor both the production of the intermediate 5-HMF and the following acetalization reaction. In the present study, a two-step strategy was involved during the conversion of fructose to HDMF: firstly, fructose was dehydrated in DMSO to form 5-HMF; secondly, 5-HMF was acetalized to HDMF after the addition of methanol with proper temperature control.

\subsubsection{The First Step: Dehydration of Fructose to 5-HMF in DMSO}

DMSO has been found to act as both an excellent solvent and a catalyst in the fructose dehydration reaction [24], but the catalytic activity of the solid acid for the dehydration is not clear, especially in the presence of methanol during the one-step biphasic experiments. Therefore, the effect of $\mathrm{SiO}_{2}-\mathrm{HNO}_{3}$ during the formation of 5-HMF from fructose was first explored in DMSO (Figure 6). The promoting effect of DMSO solvent on the dehydration of fructose to 5-HMF can be confirmed by the blank test. It is known that DMSO can promote dehydration efficiently, which could be attributed to the formation of strong acids from the thermolysis of DMSO in air such as sulfuric and methanesulfonic acids during the dehydration of fructose to 5-HMF, especially in high temperatures [25]. Moreover, DMSO would stabilize 5-HMF and suppress both the 5-HMF rehydration and the formation of condensation 
by-products because DMSO could prevent 5-HMF from rehydration to levulinic acid, formic acid, and humins by a preferential coordination of DMSO around the 5-HMF molecule, and protect the fructose molecule from reactions other than its dehydration to 5-HMF by a specific coordination of DMSO around the fructose molecule $[26,27]$.

(a)

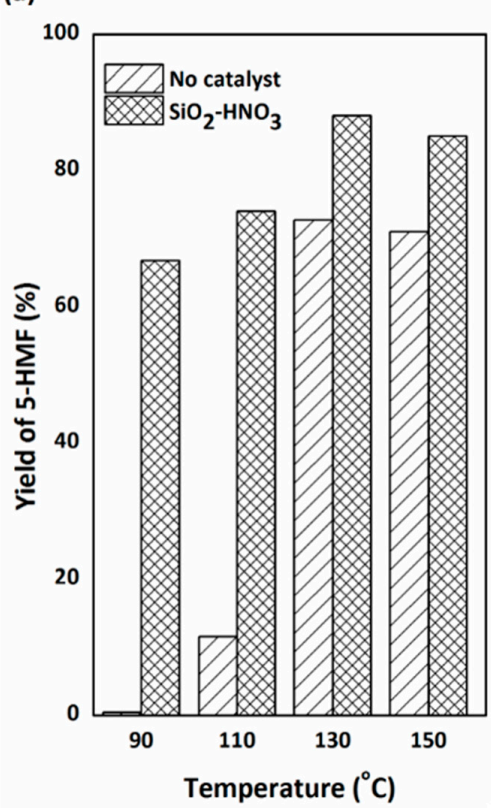

(b)

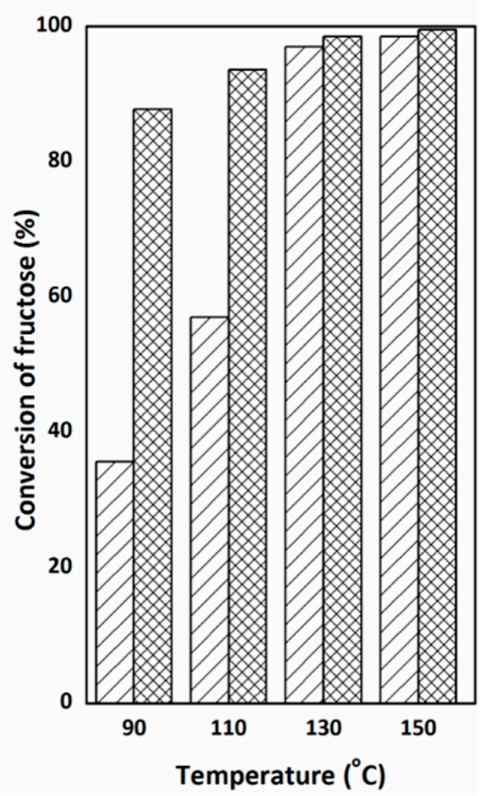

Figure 6. Effect of $\mathrm{SiO}_{2}-\mathrm{HNO}_{3}$ on fructose dehydration in DMSO at different temperatures. (a) Yield of 5-HMF, (b) Conversion of fructose. Reaction conditions: $1.37 \mathrm{mmol}$ fructose without catalyst or with $50 \mathrm{mg} \mathrm{SiO} 2-\mathrm{HNO}_{3}$ in $4.0 \mathrm{~mL}$ DMSO after $1 \mathrm{~h}$.

It was obvious that most of the fructose was converted after $1 \mathrm{~h}$, and the reaction with $\mathrm{SiO}_{2}-\mathrm{HNO}_{3}$ was always able to obtain a higher final conversion and 5- $\mathrm{HMF}$ yield than it was without $\mathrm{SiO}_{2}-\mathrm{HNO}_{3}$, demonstrating that $\mathrm{SiO}_{2}-\mathrm{HNO}_{3}$ actually accelerated the reaction and promoted the production of 5-HMF, especially at low temperatures. Further testing with longer reaction times was also conducted and no significant change in the products distribution was observed after $2 \mathrm{~h}$. However, the 5-HMF yield began to decrease at temperatures higher than $130{ }^{\circ} \mathrm{C}$, suggesting an increasing rate of the consecutive reactions, possibly due to the degradation of 5-HMF into humins.

Regarding the mechanism of the dehydration of fructose in DMSO, 1,2-enediol has been reported to be a key intermediate product [28]. In this case, the ready availability of acid sites on the $\mathrm{SiO}_{2}-\mathrm{HNO}_{3}$ catalyst were supposed to be beneficial for the formation of these intermediates. At this point, the inexpensive and readily prepared catalyst $\mathrm{SiO}_{2}-\mathrm{HNO}_{3}$ has an active effect on the dehydration of fructose when seeking to obtain similar results to costly catalysts such as Amberlyst 70 [29].

\subsubsection{The Second Step: Acetalization of 5-HMF with the Addition of Methanol}

Although 5-HMF could be obtained in pure DMSO, the yield was low due to the protic nature of methanol in the one-pot, one-step reaction. The main obstacle for the production of HDMF in the one-step reaction is the low rate of intermediate 5-HMF formation due to the interference of the protic solvent (methanol). Separating the reaction into two steps made it possible to reach an optimum $5-\mathrm{HMF}$ yield up to $88 \%$ at the first reaction, and a high concentration of methanol in the second reaction was supposed to shift the chemical equilibrium of the acetalization reaction towards the synthesis of HDMF.

Therefore, a two-step reaction was developed to promote the direct conversion of fructose towards HDMF. First, the dehydration reaction was performed at $150^{\circ} \mathrm{C}$ for $2 \mathrm{~h}$, which was the shortest time 
needed to obtain complete fructose conversion aiming at 5-HMF based on the results in the one-step reaction. Then, a certain amount of methanol was immediately added to the cooled reaction solution to convert 5-HMF into HDMF. The second step temperature was investigated and $100{ }^{\circ} \mathrm{C}$ was found to be most suitable for the production of HDMF. At $120^{\circ} \mathrm{C}$, the etherification of HDMF to MMF was favoured with a final MMF yield of ca. $15 \%$. In this way, the overall HDMF yield reached $70 \%$, which was almost twice the optimum yield obtained in the one-step design (Figure 7). It is important to note that the yield of HDMF was little without DMSO (Figure 2), indicating the favorable role of DMSO as a solvent played in the acetalization reaction, although the production of acidic degradation products in DMSO has also been reported.

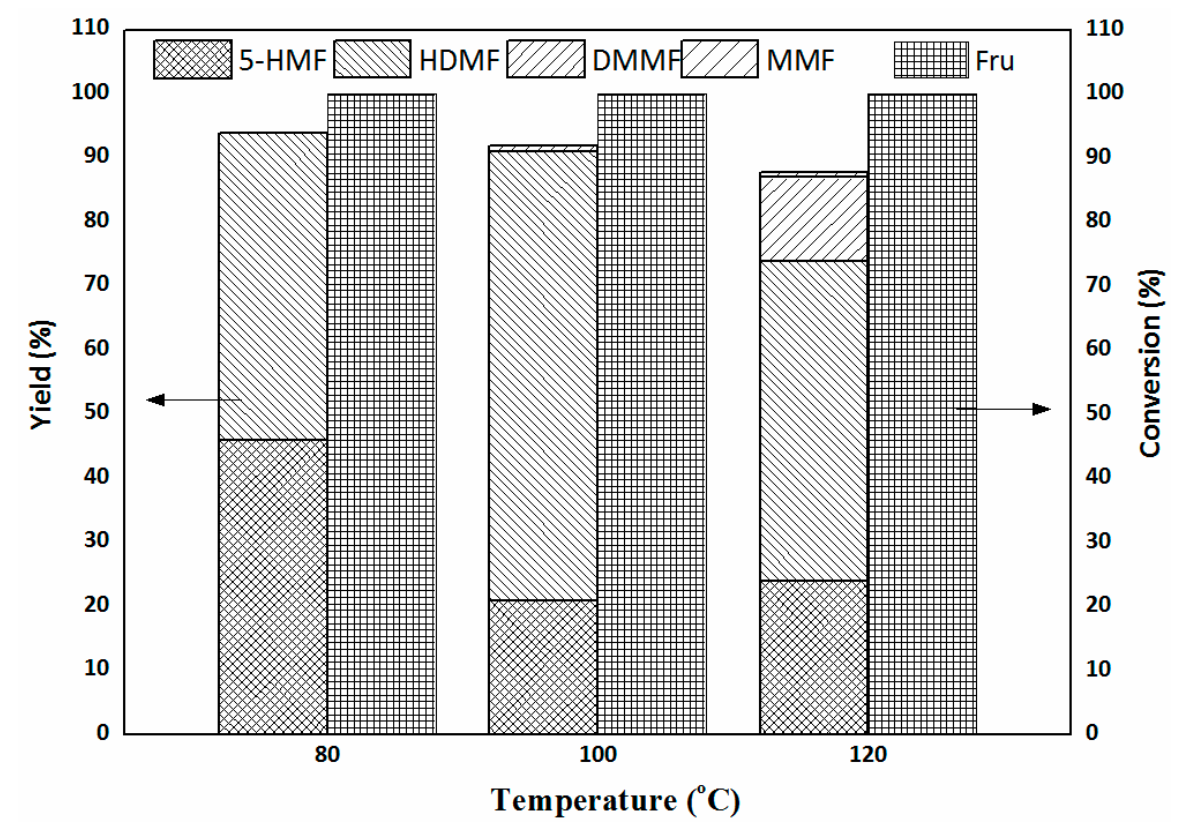

Figure 7. Effect of second-step temperature on the two-step reaction. Reaction conditions: $1.37 \mathrm{mmol}$ fructose in $4.0 \mathrm{~mL}$ DMSO at $150{ }^{\circ} \mathrm{C}$ with $50 \mathrm{mg} \mathrm{SiO} 2-\mathrm{HNO}_{3}$; after $2 \mathrm{~h}, 9.3 \mathrm{~mL}$ methanol was added and the reaction time was extended to $3 \mathrm{~h}$.

It is important to note that extended reaction periods lead to the decrease of HDMF and the increase of DMMF; hence, the implementation of an HDMF removal system to direct the equilibrium to the production of the acetal and suppress undesired further reactions is suggested.

\subsubsection{Catalyst Recycling}

The catalysts could be recycled by simple filtration and washing with acetonitrile, followed by vacuum drying. The results of the reuse study are shown in Figure 8. The catalyst $\mathrm{SiO}_{2}-\mathrm{HNO}_{3}$ proved to be recyclable, as complete fructose conversion was observed without significant loss of HDMF yield even after five consecutive runs. The yield of DMMF gradually diminished to zero as a result of the decreasing etherification of HDMF. The slight decrease in the total yield could be ascribed to the minor loss of activity of $\mathrm{SiO}_{2}-\mathrm{HNO}_{3}$ or the intensive formation of humins during the reaction. The slightly lowered BET surface area, average pore diameter, and total acid sites during each run could also result in slightly decreased activity (Table S2), indicating that $\mathrm{SiO}_{2}-\mathrm{HNO}_{3}$, as an acid catalyst, possessed excellent stability in DMSO. Thus, the $\mathrm{SiO}_{2}-\mathrm{HNO}_{3}$ catalyst can be effectively separated and reused. 


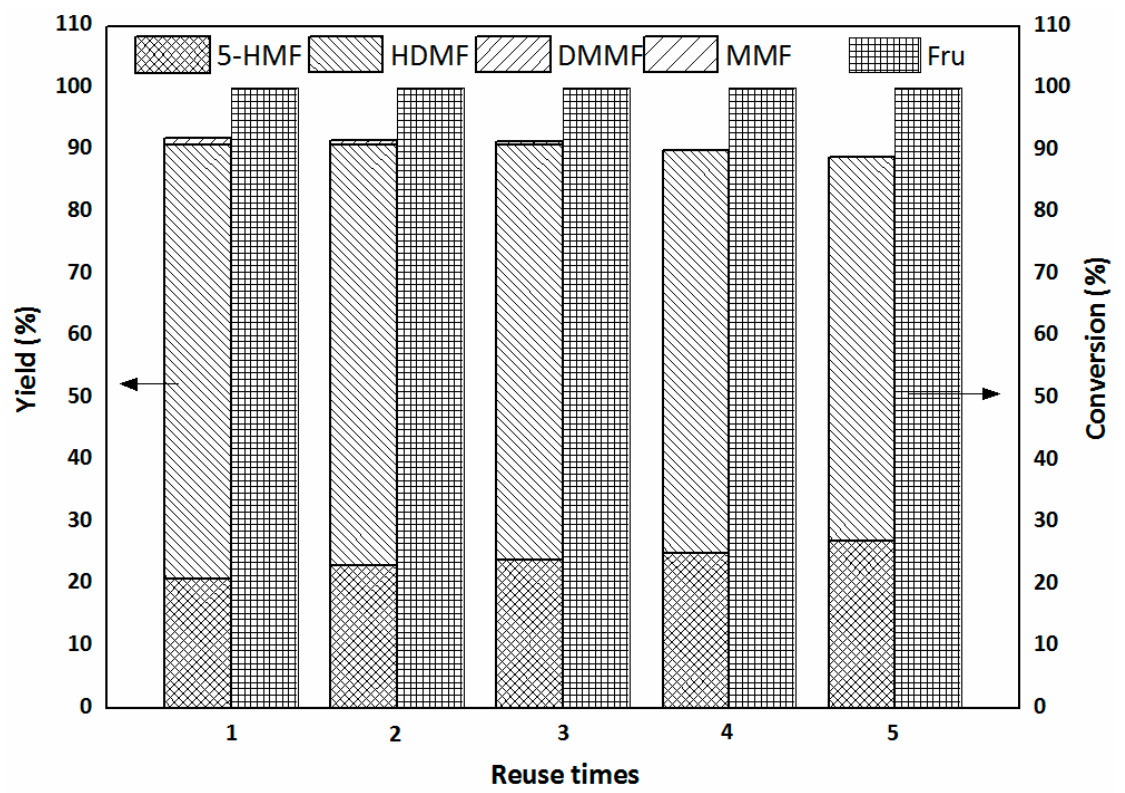

Figure 8. Results of the reuse study. Reaction conditions: $1.37 \mathrm{mmol}$ fructose in $4.0 \mathrm{~mL}$ DMSO at $150{ }^{\circ} \mathrm{C}$ with $50 \mathrm{mg} \mathrm{SiO} 2-\mathrm{HNO}_{3}$; after $2 \mathrm{~h}, 9.3 \mathrm{~mL}$ methanol was added, and the reaction time was extended to $3 \mathrm{~h}$ at $100{ }^{\circ} \mathrm{C}$.

\subsection{Proposed Mechanism for the Production of HDMF}

A plausible mechanism for the convesion of 5-HMF during the one-pot, two-step reaction is proposed in Scheme 2. The produced 5-HMF from fructose during the first step was then transformed to HDMF, DMMF, and MMF through the acetalization and etherification reactions. The dimethylacetalization of 5-HMF to HDMF was almost instantaneous in the presence of acid catalysts, and the hemiacetal 2 was supposed to be produced by protonation of $\mathbf{1}$, followed by the nucleophilic attack of methanol at the carbonyl carbon of 5-HMF [7]. Consequently, the loss of a water molecule results in the formation of an oxygen-cationic intermediate 3 , formed by combining the hydroxyl groups of 2 with the hydrogen proton of $\mathrm{SiO}_{2}-\mathrm{HNO}_{3}$. HDMF was then formed by the further nucleophilic attack of methanol. The production of DMMF from HDMF was envisioned to occur via etherification reactions with the formation of oxygen-cationic intermediate 5. The formation of MMF from DMMF was proposed to occur via a similar sequence of steps as the formation of HDMF from 5-HMF.

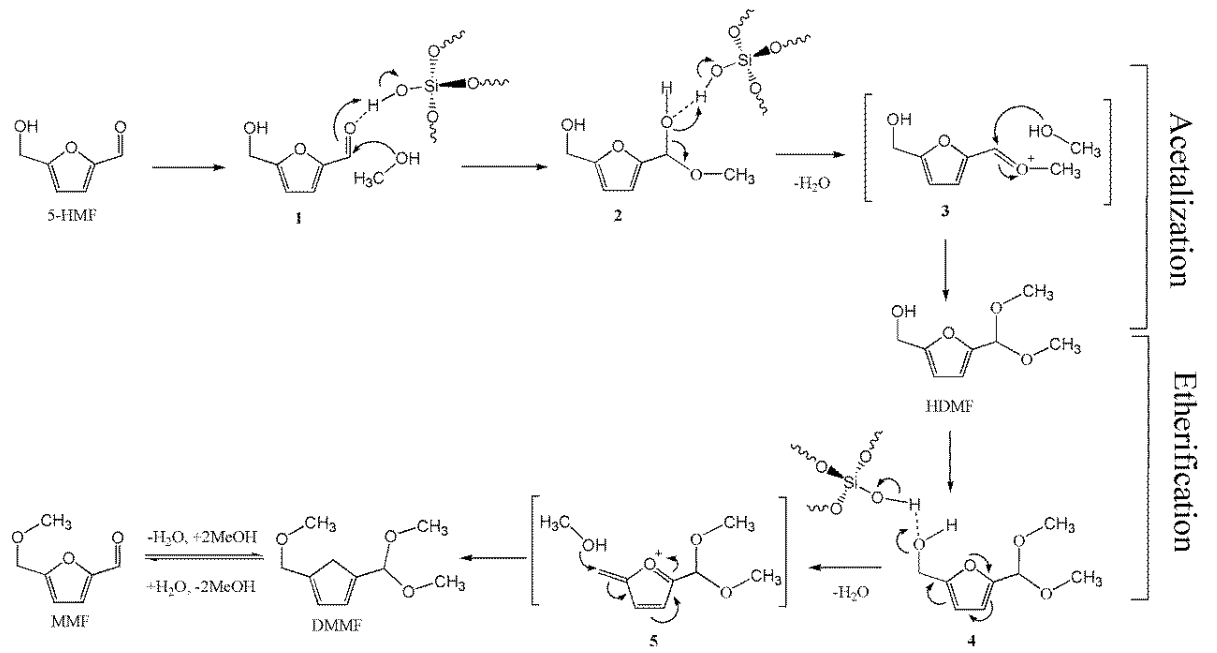

Scheme 2. Plausible mechanism pathways for the formation of HDMF, DMMF, and MMF from 5-HMF. 


\section{Experimental Section}

\subsection{Materials}

5-HMF (98\%), fructose (99\%), silica gel (300-400 mesh), and sodium hydrogen carbonate were purchased from the Aladdin Chemistry Reagent Company (Shanghai, China). N-hexane, methanol, ethanol, ethyl acetate, and DMSO were purchased from J \& K Scientific Ltd. (Beijing, China). Nitric acid (99\%) was purchased from the Alfa Aesar Company (Ward Hill, MA, USA). All reagents were used as received without further purification.

\subsection{Preparation of Catalyst}

$\mathrm{SiO}_{2}-\mathrm{HNO}_{3}$ was prepared using a literature procedure [7]. First, $30 \mathrm{~g}$ of silica gel and $300 \mathrm{~mL}$ of $1 \mathrm{M}$ nitric acid solution were mixed and stirred in a 1-L round-bottomed flask at $80^{\circ} \mathrm{C}$ for $12 \mathrm{~h}$ and then filtered. The soaking procedure was repeated three times. The resulting silica gel catalyst was then thoroughly washed with deionized water to remove the residual acid and then dried at $100{ }^{\circ} \mathrm{C}$ overnight. The silica gel-supported nitric acid was thus obtained.

The $\mathrm{SiO}_{2}-\mathrm{HNO}_{3}$ precipitated at the end of the reaction was recovered by filtration and recycled. The residues were boiled in ethanol for $2 \mathrm{~h}$ and washed with ethanol and distilled water at least three times before drying at $100{ }^{\circ} \mathrm{C}$ overnight.

\subsection{General Reaction Procedure}

In a typical reaction procedure, $1.37 \mathrm{mmol}$ of fructose, $4 \mathrm{~mL}$ of solvent (with various volume ratio of DMSO and methanol), and a certain amount of $\mathrm{SiO}_{2}-\mathrm{HNO}_{3}$ catalyst were reacted in a $50-\mathrm{mL}$ stainless-steel autoclave with a Teflon lining located in a copper heating block. The reaction temperature was detected by a thermocouple inside the vessel. After the desired reaction time, the autoclave was removed to an ice-water mixture to quench the reaction. Next, the reaction solution was neutralized by sodium hydrogen carbonate and filtered with a $0.45-\mu \mathrm{m}$ syringe filter to remove the solid catalyst and the potential solid byproducts. The samples were diluted with methanol prior to the analysis. All the data were based on at least three repeated runs.

For the one-pot, two-step reactions, $4 \mathrm{~mL}$ of DMSO was employed in the first-step reaction. When the scheduled time of the first reaction was reached, the corresponding volume of methanol was added to the cooled reaction mixture for the second-step reaction. Afterwards, the vessel was reintroduced on the heating block and heated to the temperature designated on the experiment. The constituents of the samples were analyzed by high-performance liquid chromatography (HPLC).

\subsection{Analysis Methods}

An Agilent 1200 HPLC with a Shodex IR detector and a Hypersil APS-2 column was used for the analysis of fructose. The column temperature was set at $35^{\circ} \mathrm{C}$, and water was used as the mobile phase at a flow rate of $0.2 \mathrm{~mL} / \mathrm{min}$. The concentrations of 5-HMF, HDMF, MMF, and DMMF were analyzed by HPLC (LC3000, Beijing Chuangxin Tongheng Science and Technology Co., Ltd., Beijing, China), equipped with an UV detector and a C18 column, and a mixture of deionized water/methanol $(60 / 40 \mathrm{v} / \mathrm{v})$ was used as the mobile phase with a flow rate of $1 \mathrm{~mL} / \mathrm{min}$.

Fructose and 5-HMF concentrations were determined based on external standard curves constructed using authentic standards. The derivation products of 5-HMF, including HDMF, MMF, and DMMF, were identified based on the GC-MS (Agilent 5975C, Agilent Technologies Ltd., Santa Clara, CA, USA) analysis and the results were compared to those reported in the literature. Furthermore, ${ }^{1} \mathrm{H}$ NMR was performed to confirm their formation [11,30,31]. HDMF, MMF, and DMMF were separated and purified through a column chromatography procedure based on the methods previously reported [7]. The pure products obtained were then used to develop calibration curves.

The surface area and pore size distribution of the $\mathrm{SiO}_{2}-\mathrm{HNO}_{3}$ catalyst were determined by the $\mathrm{N}_{2}$ adsorption method through a Brunauer-Emmett-Teller (BET) surface area analyzer 
(SORPTOMATIC 1990, Thermo Electron Corporation, Waltham, MA, USA). The total acid sites were measured by the temperature-programmed desorption of ammonia $\left(\mathrm{NH}_{3}\right.$-TPD) (AutoChem 2910, Micromeritics Instrument Corp., Norcross, GA, USA). The structure of $\mathrm{SiO}_{2}-\mathrm{HNO}_{3}$ was characterized by Fourier transform infrared spectroscopy (FTIR) (TENSOR 27, Bruker, Billerica, MA, USA).

\subsection{Calculation of Yield and Conversion}

The definitions of fructose conversion and product yields are as follows:

Conversion $=(1$-moles of fructose in product $/$ initial moles of fructose $) \times 100 \%$

Yield $=($ moles of product produced $/$ initial moles of fructose $) \times 100 \%$

Selectivity $=($ moles of product produced $/$ moles of fructose converted $) \times 100 \%$

\section{Conclusions}

The acid-catalyzed dehydration and acetalization of fructose in alcoholic/DMSO biphasic solvents provides routes to HDMF, DMMF, and MMF, which are potential biodiesel fuels. The maximum yield of $\mathrm{HDMF}(77 \%)$ was obtained by the direct reaction of $5-\mathrm{HMF}$ with methanol over $\mathrm{SiO}_{2}-\mathrm{HNO}_{3}$. It is necessary to use fructose as a substrate in a biphasic $\mathrm{DMSO} /$ methanol system to reduce costs. The optimal HDMF yield was 34\% under optimized reaction conditions. The influence of the protic solvent methanol possibly resulted in a low yield of 5-HMF, and an alternative two-step arrangement of the reaction was designed, leading to an HDMF yield of up to $70 \%$ with the complete conversion of fructose. The $\mathrm{SiO}_{2}-\mathrm{HNO}_{3}$ catalyst could be recycled by a simple process at the end of the reaction and reused several times without a significant loss of activity. A plausible reaction mechanism for the conversion of 5-HMF to HDMF was proposed. The excellent performance of $\mathrm{SiO}_{2}-\mathrm{HNO}_{3}$ catalysts in the one-pot, two-step system encourages us to further investigate the possibility of the current system for the direct conversion of other carbohydrates to HDMF.

Supplementary Materials: The following are available online at www.mdpi.com/2073-4344/7/8/237/s1. Table S1: Characterization of the catalysts based on silica gel. Table S2: Characterization of the reused catalysts $\mathrm{SiO}_{2}-\mathrm{HNO}_{3}$. Table S3: Rate constants (k) of 5-HMF conversion with methanol at different reaction temperatures ${ }^{\mathrm{a}}$. Table S4: Kinetic parameters of 5-HMF conversion with methanol. Table S5: Rate constants (k) of fructose conversion at different reaction temperatures in the DMSO/methanol system a. Table S6: Kinetic parameters of the fructose conversion in the DMSO/methanol system. Figure S1: XRD analysis of $\mathrm{SiO}_{2}-\mathrm{HNO}_{3}$. Figure S2: ${ }^{29} \mathrm{Si} \mathrm{NMR}$ analysis of $\mathrm{SiO}_{2}-\mathrm{HNO}_{3}$. Figure S3: FTIR spectra of $\mathrm{SiO}_{2}-\mathrm{HNO}_{3}$. Figure S4: Effect of acid-treatment on the activity of the silica gel in the two-step reaction. Reaction conditions: $1.37 \mathrm{mmol}$ fructose in $4.0 \mathrm{~mL}$ DMSO at $150{ }^{\circ} \mathrm{C}$ with $50 \mathrm{mg} \mathrm{SiO} 2-\mathrm{HNO}_{3}$ or $\mathrm{SiO}_{2}$; after $2 \mathrm{~h}, 9.3 \mathrm{~mL}$ methanol was added and the reaction time was extended to $3 \mathrm{~h}$ at $100^{\circ} \mathrm{C}$.

Acknowledgments: This study was sponsored by the National Natural Science Foundation of China under grant number 21476021.

Author Contributions: Chongpin Huang and Xincheng Wang conceived and designed the experiments; Guo Qiu performed the experiments; Guo Qiu and Xincheng Wang analyzed the data; Yingxia Li and Biaohua Chen contributed reagents/materials/analysis tools; Guo Qiu, Xincheng Wang and Chongpin Huang wrote the paper.

Conflicts of Interest: The authors declare no competing financial interest.

\section{References}

1. Van Putten, R.-J.; van der Waal, J.C.; de Jong, E.; Rasrendra, C.B.; Heeres, H.J.; de Vries, J.G. Hydroxymethylfurfural, a versatile platform chemical made from renewable resources. Chem. Rev. 2013, 113, 1499-1597. [CrossRef] [PubMed]

2. Baliban, R.C.; Elia, J.A.; Floudas, C.A.; Xiao, X.; Zhang, Z.; Li, J.; Cao, H.; Ma, J.; Qiao, Y.; Hu, X. Thermochemical conversion of duckweed biomass to gasoline, diesel, and jet fuel: Process synthesis and global optimization. Ind. Eng. Chem. Res. 2013, 52, 11436-11450. [CrossRef]

3. Ranoux, A.; Djanashvili, K.; Arends, I.W.C.E.; Hanefeld, U. 5-Hydroxymethylfurfural synthesis from hexoses is autocatalytic. ACS Catal. 2013, 3, 760-763. [CrossRef] 
4. Roylance, J.J.; Choi, K.S. Electrochemical reductive biomass conversion: Direct conversion of 5-hydroxymethylfurfural (HMF) to 2,5-hexanedione (HD) via reductive ring-opening. Green Chem. 2016, 18, 2956-2960. [CrossRef]

5. Agirre, I.; Güemez, M.B.; Ugarte, A.; Requies, J.; Barrio, V.L.; Cambra, J.F.; Arias, P.L. Glycerol acetals as diesel additives: Kinetic study of the reaction between glycerol and acetaldehyde. Fuel Process. Technol. 2013, 116, 182-188. [CrossRef]

6. Cui, W.; Qi, M.; Li, X.; Huang, S.; Zhou, S.; Weng, J. Electrospun fibers of acid-labile biodegradable polymers with acetal groups as potential drug carriers. Int. J. Pharm. 2008, 361, 47-55. [CrossRef] [PubMed]

7. Balakrishnan, M.; Sacia, E.R.; Bell, A.T. Etherification and reductive etherification of 5-(hydroxymethyl) furfural: 5-(alkoxymethyl) furfurals and 2,5-bis (alkoxymethyl) furans as potential bio-diesel candidates. Green Chem. 2012, 14, 1626. [CrossRef]

8. Lai, L.; Zhang, Y. The production of 5-hydroxymethylfurfural from fructose in isopropyl alcohol: A green and efficient system. ChemSusChem 2011, 4, 1745-1748. [CrossRef] [PubMed]

9. Arias, K.S.; Climent, M.J.; Corma, A.; Iborra, S. Biomass-Derived Chemicals: Synthesis of Biodegradable Surfactant Ether Molecules from Hydroxymethylfurfural. ChemSusChem 2014, 7, 210-220. [CrossRef] [PubMed]

10. Arias, K.S.; Al-Resayes, S.I.; Climent, M.J.; Corma, A.; Iborra, S. From Biomass to Chemicals: Synthesis of Precursors of Biodegradable Surfactants from 5-Hydroxymethylfurfural. ChemSusChem 2013, 6, $123-131$. [CrossRef] [PubMed]

11. Hu, X.; Gunawan, R.; Mourant, D.; Lievens, C.; Li, X.; Zhang, S.; Chaiwat, W.; Li, C.-Z. Acid-catalysed reactions between methanol and the bio-oil from the fast pyrolysis of mallee bark. Fuel 2012, 97, 512-522. [CrossRef]

12. Hu, X.; Li, C.-Z. Levulinic esters from the acid-catalysed reactions of sugars and alcohols as part of a bio-refinery. Green Chem. 2011, 13, 1676. [CrossRef]

13. Najafi, M.; Rostamian, R.; Rafati, A.A. Chemically modified silica gel with thiol group as an adsorbent for retention of some toxic soft metal ions from water and industrial effluent. Chem. Eng. J. 2011, 168, 426-432. [CrossRef]

14. Kaur, M.; Sharma, S.; Bedi, P.M.S. Silica supported Brönsted acids as catalyst in organic transformations: A comprehensive review. Chin. J. Catal. 2015, 36, 520-549. [CrossRef]

15. Xu, S.; Yan, X.; Bu, Q.; Xia, H. Highly efficient conversion of carbohydrates into 5-hydroxymethylfurfural using the bi-functional $\mathrm{CrPO}_{4}$ catalyst. RSC Adv. 2016, 6, 8048-8052. [CrossRef]

16. Yu, Y.; Hu, C.; Abu-Omar, M.M. Conversion of glucose into furans in the presence of $\mathrm{AlCl}_{3}$ in an ethanol-water solvent system. Bioresour. Technol. 2012, 116, 190-194.

17. Karinen, R.; Vilonen, K.; Niemelä, M. Biorefining: Heterogeneously catalyzed reactions of carbohydrates for the production of furfural and hydroxymethylfurfural. ChemSusChem 2011, 4, 1002-1016. [CrossRef] [PubMed]

18. Kimura, H.; Nakahara, M.; Matubayasi, N. Solvent effect on pathways and mechanisms for D-fructose conversion to 5-hydroxymethyl-2-furaldehyde: In situ ${ }^{13}$ C NMR study. J. Phys. Chem. A 2013, 117, 2102-2113. [CrossRef] [PubMed]

19. Liu, J.; Tang, Y.; Wu, K.; Bi, C.; Cui, Q. Conversion of fructose into 5-hydroxymethylfurfural (HMF) and its derivatives promoted by inorganic salt in alcohol. Carbohydr. Res. 2012, 350, 20-24. [CrossRef] [PubMed]

20. Bicker, M.; Kaiser, D.; Ott, L.; Vogel, H. Dehydration of D-fructose to hydroxymethylfurfural in sub-and supercritical fluids. J. Supercrit. Fluids 2005, 36, 118-126. [CrossRef]

21. Shimizu, K.I.; Uozumi, R.; Satsuma, A. Enhanced production of hydroxymethylfurfural from fructose with solid acid catalysts by simple water removal methods. Catal. Commun. 2009, 10, 1849-1853. [CrossRef]

22. Wang, H.; Deng, T.; Wang, Y.; Qi, Y.; Hou, X.; Zhu, Y. Efficient catalytic system for the conversion of fructose into 5-ethoxymethylfurfural. Bioresour. Technol. 2013, 136, 394-400. [CrossRef] [PubMed]

23. Qu, Y.; Huang, C.; Song, Y.; Zhang, J.; Chen, B. Efficient dehydration of glucose to 5-hydroxymethylfurfural catalyzed by the ionic liquid, 1-hydroxyethyl-3-methylimidazolium tetrafluoroborate. Bioresour. Technol. 2012, 121, 462-466. [CrossRef] [PubMed]

24. Rosatella, A.A.; Simeonov, S.P.; Frade, R.F.M.; Afonso, C.A.M. 5-Hydroxymethylfurfural (HMF) as a building block platform: Biological properties, synthesis and synthetic applications. Green Chem. 2011, 13, 754-793. [CrossRef] 
25. Laugel, C.; Estrine, B.; Le Bras, J.; Hoffmann, N.; Marinkovic, S.; Muzart, J. NaBr/DMSO-Induced Synthesis of 2,5-Diformylfuran from Fructose or 5-(Hydroxymethyl) furfural. ChemCatChem 2014, 6, 1195-1198. [CrossRef]

26. Despax, S.; Maurer, C.; Estrine, B.; Bras, J.L.; Hoffmann, N.; Marinkovic, S.; Muzart, J. Fast and efficient DMSO-mediated dehydration of carbohydrates into 5-hydroxymethylfurfural. Catal. Commun. 2014, 51, 5-9. [CrossRef]

27. Mushrif, S.H.; Caratzoulas, S.; Vlachos, D.G. Understanding solvent effects in the selective conversion of fructose to 5-hydroxymethyl-furfural: A molecular dynamics investigation. Phys. Chem. Chem. Phys. 2012, 14, 2637-2644. [CrossRef] [PubMed]

28. Antal, M.J., Jr.; Mok, W.S.L.; Richards, G.N. Mechanism of formation of 5-(hydroxymethyl)-2-furaldehyde from D-fructose and sucrose. Carbohydr. Res. 1990, 199, 91-109. [CrossRef]

29. Morales, G.; Melero, J.A.; Paniagua, M.; Iglesias, J.; Hernández, B.; Sanz, M. Sulfonic acid heterogeneous catalysts for dehydration of C 6-monosaccharides to 5-hydroxymethylfurfural in dimethyl sulfoxide. Chin. J. Catal. 2014, 35, 644-655. [CrossRef]

30. Hu, X.; Lievens, C.; Larcher, A.; Li, C.Z. Reaction pathways of glucose during esterification: Effects of reaction parameters on the formation of humin type polymers. Bioresour. Technol. 2011, 102, 10104-10113. [CrossRef] [PubMed]

31. Cottier, L.; Descotes, G.; Soro, Y. Synthesis of Acetylated Ranunculin Diastereoisomers and $\delta$-Glucosyloxy- $\gamma$-Oxo Esters from $\alpha$ or $\beta$ Glucosylmethylfurfural. J. Carbohydr. Chem. 2005, 24, 55-71. [CrossRef]

(C) 2017 by the authors. Licensee MDPI, Basel, Switzerland. This article is an open access article distributed under the terms and conditions of the Creative Commons Attribution (CC BY) license (http://creativecommons.org/licenses/by/4.0/). 